\title{
Controlled Electrochemical Li Cycling in a TEM to Observe Li Morphology Evolution
}

Katherine L. Jungjohann ${ }^{1}$, Katharine L. Harrison ${ }^{2}$, Andrew J. Leenheer ${ }^{1}$, Nathan T. Hahn ${ }^{2}$, and Kevin R. Zavadil $^{2}$

1. Center for Integrated Nanotechnologies, Sandia National Laboratories, Albuquerque, New Mexico 87185

2. Sandia National Laboratories, Albuquerque, New Mexico 87185

To meet the increased demand for high power energy storage for grid and transportation applications, a stable highly efficient Li metal battery electrode is being investigated. The system is dependent on the formation of a solid electrolyte interphase that is capable of suppressing Li dendrite formation, which is the limiting characteristic that prevents application of high capacity Li metal anodes in current lithium ion batteries (LIBs). The SEI layer that is formed between the electrolyte and the Li metal electrode is dependent on the breakdown of the $\mathrm{Li}$ containing electrolyte. We investigated lithium bis(fluorosulfonyl)imide (LiFSI) in dimethoxyethane (DME) for suppressed Li dendrite formation [1]. This electrolyte is targeted for stable stripping of lithium at current densities up to $10 \mathrm{~mA} / \mathrm{cm}^{2}$ and Coulombic efficiencies above $99.1 \%$. The morphological evolution of the Li deposition and stripping on copper electrodes was monitored using quantitative in situ scanning transmission electron microscopy (STEM) in a custom fabricated electrochemical cell.

Electrochemical control within a TEM was pioneered by F. Ross in 2003 using MEMS technology [2] to study electrochemical processes of volatile electrolytes at nanoscale resolution [3]. The Center for Integrated Nanotechnologies has successfully designed a microfabricated liquid cell that can operate with picoampere current control over 10 ultramicroelectrodes while imaging within a TEM [4]. The electrodes are patterned onto a $50 \mathrm{~nm} \mathrm{SiN}$ xembrane window, with a fluid gap around $150 \mathrm{~nm}$. This design allows for multiple experiments to be performed within the same closed cell, where we studied varied current densities and beam conditions under identical environmental conditions. The electrochemical platform was custom patterned using e-beam lithography to deposit $\mathrm{Cu}$ leads coated with a passivation layer of $\mathrm{Al}_{2} \mathrm{O}_{3}$ and $\mathrm{SiO}_{2}$, where $\sim 1 \mathrm{~m}^{2} \mathrm{Cu}$ electrodes and large $>100 \mu \mathrm{m}^{2} \mathrm{Si}$ electrodes were exposed, Figure 1.

The patterned electrochemical platform was loaded within a He filled glove box with 4M LiFSI in DME, then epoxy coated to hermetically seal the cell. $\mathrm{Li}_{\mathrm{x}} \mathrm{Si}$ quasireference electrodes were prepared within the TEM by applying $100 \mathrm{pA}$ between two Si electrodes, and lithiation of the cathodically polarized Si could be observed by decreased contrast in the Si electrode. The cell was then set up as a three electrode experiment with $\mathrm{Cu}$ working electrodes, a $\mathrm{Si}$ counter electrode, and a $\mathrm{Li}_{\mathrm{x}} \mathrm{Si}$ quasireference electrode. Three $\mathrm{Cu}$ electrodes were cycled through 10 lithiation deposition and stripping steps at $2.25 \mathrm{~mA} / \mathrm{cm}^{2}$. Each electrode was exposed to different total beam exposures of $0,<4 \mathrm{e}^{-} / \AA^{2}$, and $<21 \mathrm{e}^{-} / \AA^{2}$ of STEM imaging during over the 10 cycles. The morphology of the deposited Li exhibited dendrite formation at each of the tested beam conditions. At the highest beam exposure, shown in Figure 2, many Li structures formed including dendrites, porous deposits, hexagonal grains, and small dense deposits. At this scale, Li prefers the formation of many nucleation sites in contrast to one single grain. These results provide an understanding of the deposit morphology at each step in addition to the final deposit structure which is typically analysed after coin cell studies [5]. 


\section{References:}

[1] R. Miao et al, J. Power Sources 271 (2014), p. 291.

[2] M. J. Williamson et al, Nat. Mater. 2 (2003), p. 532.

[3] F. Wu and N. Yao, Nano Energy 11 (2015), p. 196.

[4] A. J. Leenheer et al, J. Microelectromech. S. 99 (2015), 10.1109/JMEMS.2014.2380771.

[5] This work was performed at the Center for Integrated Nanotechnologies (CINT), a U.S. DOE Office of Basic Energy Sciences (BES) user facility. Sandia National Laboratories is a multiprogram laboratory managed and operated by Sandia Corporation, a wholly-owned subsidiary of Lockheed Martin Corporation, for the U.S. DOE's National Nuclear Security Administration under contract DEAC0494AL85000. Portions of this work were funded by the Nanostructures for Electrical Energy Storage, an Energy Frontier Research Center funded by the U.S. DOE, Office of Science. Other portions were funded by the Joint Center for Energy Storage Research, an Energy Innovation Hub funded by the U.S. DOE, Office of Science.

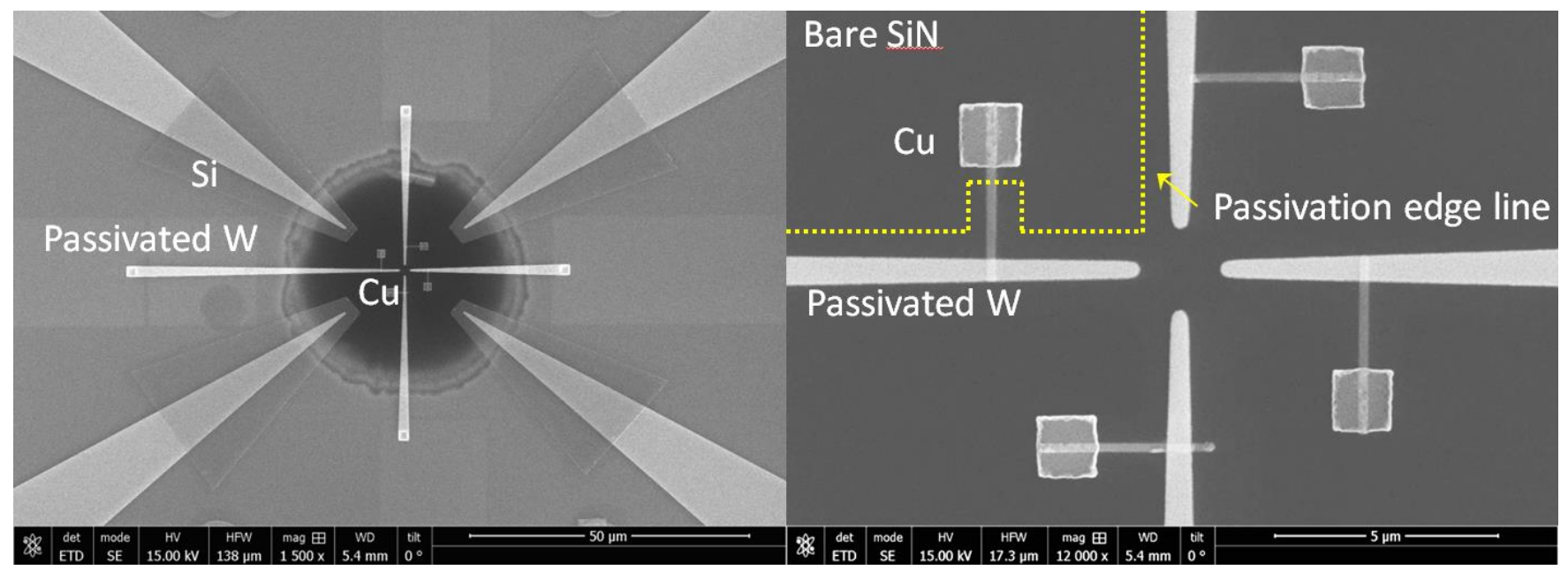

Figure 1. Custom patterned electrochemical platform containing tungsten electrodes, passivated then patterned with four $\mathrm{Cu}$ working electrodes, and four $\mathrm{Si}$ electrodes to be used as $\mathrm{Li}_{\mathrm{x}} \mathrm{Si}$ quasireference and counter electrodes.

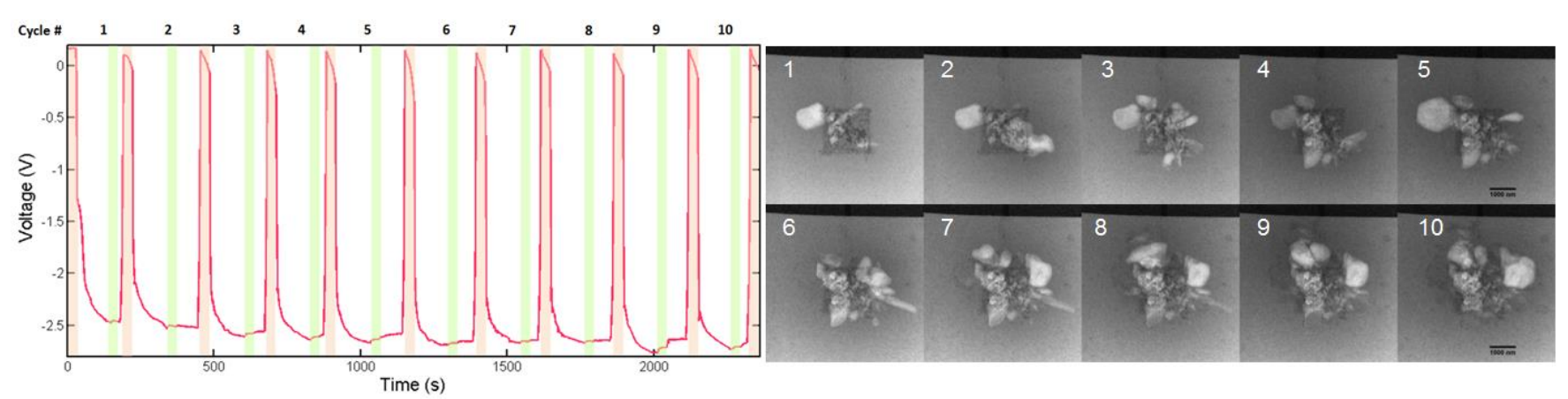

Figure 2. Ten Li deposition/stripping cycles, displaying the measured voltage over time under galvanostatic control, and representative bright field STEM images at the final Li deposition step for each cycle where Li deposits appear as bright structures. Shaded regions in data represent open circuit steps between cycles. Scale bar represents $1 \mu \mathrm{m}$. 